\title{
Study of the performance of Micromegas detectors in magnetic field
}

\author{
Sampsonidis Dimitrios ${ }^{1, a}$, On behalf of the ATLAS Muon Collaboration \\ ${ }^{1}$ Aristotle University of Thessaloniki, Thessaloniki 54124, Greece
}

\begin{abstract}
Resistive Micromegas (MICRO MEsh GAseous Structure) detectors have been chosen by the ATLAS collaboration at LHC for the high luminosity upgrade, due to their capability to maintain full efficiency and high spatial resolution at high occupancy, for tracking muons in the forward region of the detector. The Inner Muon Station, in the high-rapidity region, the so called New Small Wheel (NSW), will be composed of micromegas detectors that will have to maintain good performance in the presence of magnetic field of up to about $0.3 \mathrm{~T}$. The response of micromegas detectors is affected by the magnetic field, where the deflection of the drift electrons is described by the Lorentz angle, resulting in a bias in the reconstructed track position. Several test-beam campaigns have been performed to test the behaviour of small size resistive micromegas prototypes $\left(10 \times 10 \mathrm{~cm}^{2}\right)$ in magnetic fields up to $1 \mathrm{~T}$, using high momentum muon and hadron beams at CERN. These studies are performed in order to validate the capability of the chambers to provide unbiased tracks in the NSW conditions. Measurements of the Lorentz angle and drift velocity as a function of the magnetic field are presented and both are compared to expectations based on Garfield-Magboltz simulations. Several methods to correct the position bias are applied, based on the chamber configuration or on the knowledge of the local value of the magnetic field. The results of these studies are presented together with an overall discussion of the Micromegas tracking capability in magnetic field.
\end{abstract}

\section{Introduction}

The LHC will be upgraded again during the second long shutdown (LS2) in 2018 [1]. After the LS2 the accelerator luminosity will increased to $2 \times 10^{34} \mathrm{~cm}^{-2} \mathrm{~s}^{-1}$, allowing ATLAS to collect approximately $100 \mathrm{fb}^{-1} /$ year of data. The ATLAS detector [2], in order to benefit from the expected high luminosity performance, needs to be upgraded following the same schedule as the LHC. The Phase-I upgrade of the ATLAS muon spectrometer [1] focuses on the end-cap region, namely on the replacement of the inner station of the ATLAS muon end-cap system (Small Wheel, $\mathrm{SW}$ ). The New Small Wheel (NSW) will have to operate in a high background radiation region while, providing muon tracks with high precision, as well as information for the Level-1 trigger [3].

The performance criteria demand for the precision track reconstruction a spatial resolution of about $100 \mu \mathrm{m}$ and for the Level-1 trigger, on-line track segments with an angular resolution of approximately $1 \mathrm{mrad}$.

Two detector technologies have been chosen by the ATLAS collaboration for the NSW: small-strip Thin Gap Chambers (sTGC) as the primary trigger and Micromegas $(\mathrm{MM})$ as the primary precision tracker [4].

With the proposed NSW the ATLAS muon system will maintain its excellent muon tracking in its entire acceptance region, even at the highest luminosities expected at the LHC . At the same time the Level-1 low $p_{T}$ (typically

\footnotetext{
a e-mail: Dimitrios.Sampsonidis@ @ern.ch
}

$p_{T}>20 \mathrm{GeV}$ ) single muon trigger rate, will be kept at an acceptable level.

\section{The New Small Wheel Layout}

The NSW, following the present structure of the Muon Spectrometer, is divided in sixteen sectors, eight large and eight small sectors. Each sector comprises sixteen layers of trigger and precision tracking detectors, namely sTGCs and MM covering a total area of $1200 \mathrm{~m}^{2}$. Fig. 1 shows the MM segmentation in the large and the small sectors of the NSW [5].
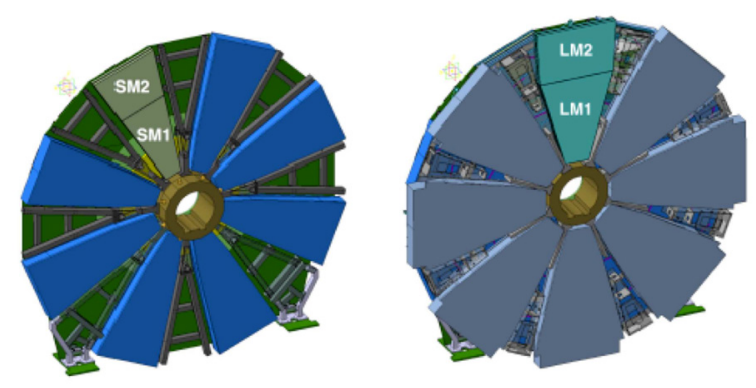

Figure 1. The two sides of the NSW showing the micromegas chambers segmentation. The Small sector (left) has two types of chambers, the SM1 and SM2 while the Large sector (right) has the LM1 and LM2 chambers 
For both technologies, each module is a multiplet of four active layers each (hence quadruplets) and is arranged in the following order: STGC-MM-MM-sTGC. The arrangement of the sTGC and MM modules will maximize the distance between the two sTGCs multilayers for improved track segment angular resolution at the trigger level. In total sixteen points will be measured for each track crossing the NSW. The choice of eight planes per detector will provide a robust, fully functional detector system over its whole lifetime.

In the radial direction the MM chambers are segmented in two. For the large sectors there are LM1 and LM2 while for the small sectors SM1 and SM2 respectively, where 1 and 2 refer to the small and the large radii.

\section{The Micromegas chambers}

The MM chambers have trapezoidal shape, sizes between 2 and $3 \mathrm{~m}^{2}$ and they are arranged in quadruplets in order to provide four independent points.

Within a quadruplet the four MM layers are grouped in two pairs. In each pair the detectors are mounted backto-back onto stiffening panels with internal structure of aluminium honeycomb as shown in Fig. 2. The readout strips in the first pair of detector layers are parallel to the base of the trapezoid in order to measure the precision coordinate ( $\eta$-strips). The readout strips, in the other two layers, are inclined by \pm 1.5 (stereo strips) with respect to the $\eta$ - strips, to provide the second coordinate (azimuthal) measurement. The strip pitch for the small sectors will be $425 \mu \mathrm{m}$ while for the large ones $450 \mu \mathrm{m}$ resulting in about 2x106 readout channels in total for the MM system of the NSW.

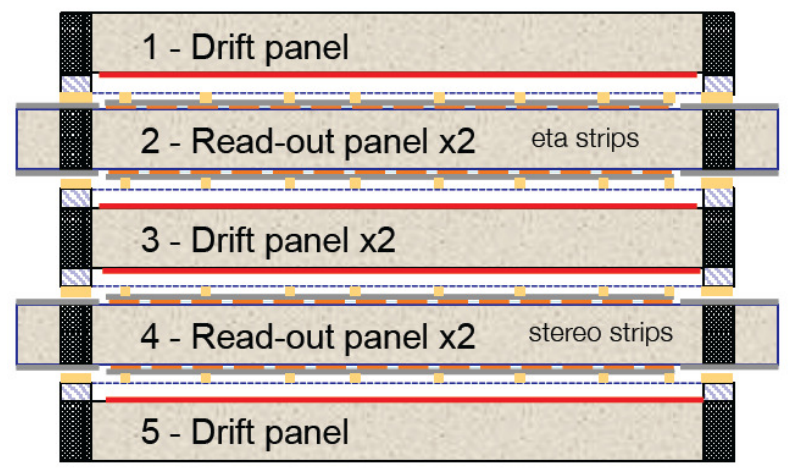

Figure 2. The arrangement of the MM layers within a quadruplet. The drift and readout electrodes are mounted back-to-back onto stiffening panels.

\section{The Micromegas detector}

The MM detector has been developed in the 1990's [4]. The principles of operation of the MM chambers chosen for the NSW are shown in Fig. 3, as well as the baseline parameters of the ATLAS-NSW chambers.

It consists of planar (Drift) electrode, a gas gap of a five millimetres thickness acting as conversion and drift region, and a fine metallic mesh at $128 \mu \mathrm{m}$ distance from the readout electrode, creating the amplification region. The readout strips are covered by a layer of resistive strips on top of a thin insulator. In such a way the MM is protected from the sparks and able to operate in high rate environment [6]. The signals are capacitively coupled to the readout electrode that is not anymore directly exposed to the charge created in the amplification region. For the ATLAS NSW, the mesh has been grounded and positive HV was applied to the resistive strips.

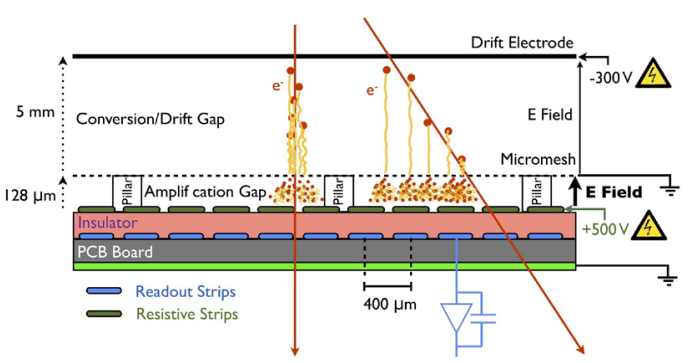

Figure 3. Schematic view of the Micromegas detector (not to scale) and the principles of operation.

When the ionising particle traverses the active volume of the micromegas it causes ionization of the gas. The electrons produced by the ionization drift towards the mesh due to the electric field of a few hundred $\mathrm{V} / \mathrm{cm}$ in the drift region. Having an electric field of $40-50 \mathrm{kV} / \mathrm{cm}$ in the amplification region the mesh is almost transparent to the electrons. The electron avalanche takes place in the amplification region above the strips. The drift of the electrons in the conversion gap is a slow process and depends on the gas Ar: $\mathrm{CO}_{2}$ (93:7), the drift distance and the drift field takes several tens of nanoseconds while the amplification process happens in a fraction of a nanosecond, resulting in a fast pulse of electrons on the readout strip.

The MM will provide spatial resolution better than $100 \mu \mathrm{m}$ independent of track incident angle. The performance of MM detectors was studied with prototypes of various sizes with particles in beams. These studies are based on the properties of the reconstructed clusters. The cluster is defined as a group of fired strips with charge above a threshold. The cluster position is evaluated utilizing a combination of two algorithms. For small incident angles, a simple cluster centroid method is employed where the position is calculated as the average of strip position weighted by the strip charge. For larger incident angles, the microTime-Projection-Chamber ( $\mu$ TPC) method is used. This method exploits the single strip time information. A track is reconstructed inside the drift gap by converting the measured drift time of charges arriving on individual strips to the position from which the drift electrons originate based on the drift velocity. The cluster position is calculated as the interpolation of the track in the middle of the drift gap. The $\mu$ TPC method has been successfully applied in several test-beam experiments at CERN. 


\section{Micromegas in a Magnetic Field}

The MM chambers of the NSW will be exposed to stray magnetic fields from the barrel and end-cap toroids with field strengths of up to about $0.3 \mathrm{~T}$ with different orientations with respect to the chamber planes but with a sizable component orthogonal to the MM electric field. The effect of the magnetic field on the detector operation has been studied with test beam data and simulations.

Fig. 4 shows the $\phi$ component of the magnetic filed in the ATLAS detector at a distance $\mathrm{z}=7800 \mathrm{~mm}$ from the interaction point [[7].

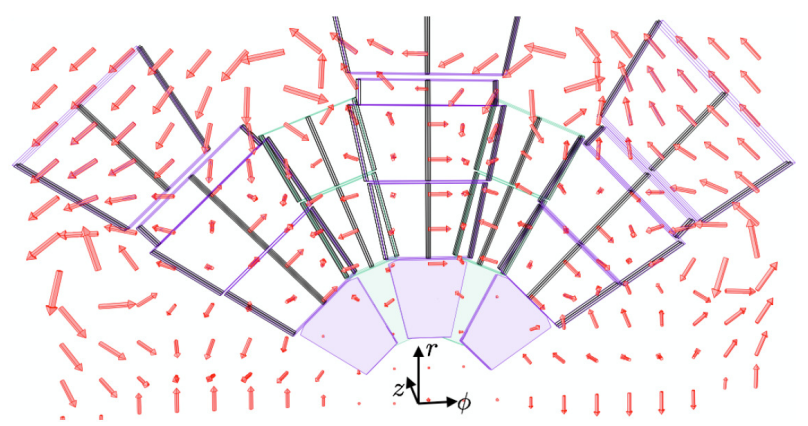

Figure 4. The $\phi$ component of the magnetic field at a distance $\mathrm{z}=7800 \mathrm{~mm}$ from the interaction point. The currently installed detectors of the Small Wheel (MDT, CSC) are displayed for reference. The plot has been created with Persint ATLAS software [8].

The electron drift velocity and the Lorentz angle as a function of the electric field for the gas mixture, $\mathrm{Ar}: \mathrm{CO}_{2}$ (93:7), were simulated under different magnetic fields using the Garfield [8] and the results are shown in Fig. 5(a) and 5(b) respectively.
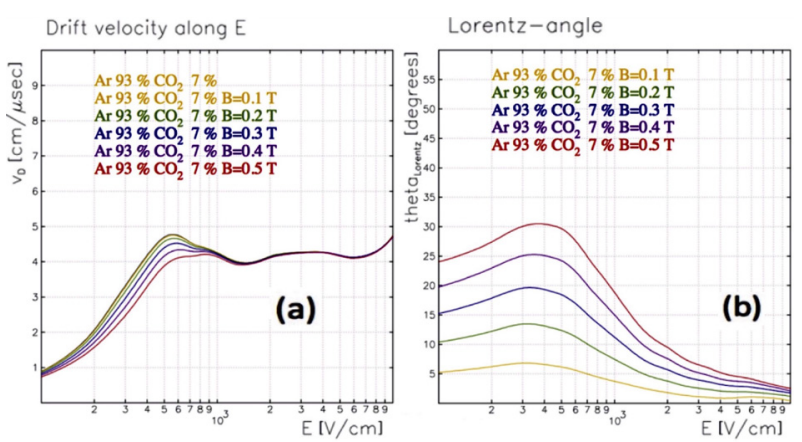

Figure 5. Drift velocity along the electric field (a) and Lorentz angle (b) as a function of the drift field for different values of the magnetic field.

The effect of the magnetic field on the detector operation has been studied with test beam data and simulations. Figure 6 illustrates the effect of the magnetic field on a MM chamber. The drift direction of the ionization electrons is tilted with respect to the electric field direction by the Lorentz angle $\theta_{L} ; \tan \theta_{L}$ is proportional to the magnetic field intensity $\mathrm{B}$. The tilt of the drift direction gives a sizable shift $(\mathrm{x})$ of the reconstructed hit position (Fig. 6a).
This shift affects both centroid and $\mu \mathrm{TPC}$ reconstruction and reaches values up to $400 \mu \mathrm{m}$. Singular configurations are expected when $\theta=\theta_{L}, \theta$ being the inclination angle of the track (Fig. 6b).

In this configuration the ionization cluster is confined to a very small number of strips and the centroid method can be applied. On the other hand, when $\theta$ and $\theta_{L}$ have different signs, the ionization cluster is spread over a larger number of strips. A combination algorithm can be applied to have a constant resolution through all configurations.
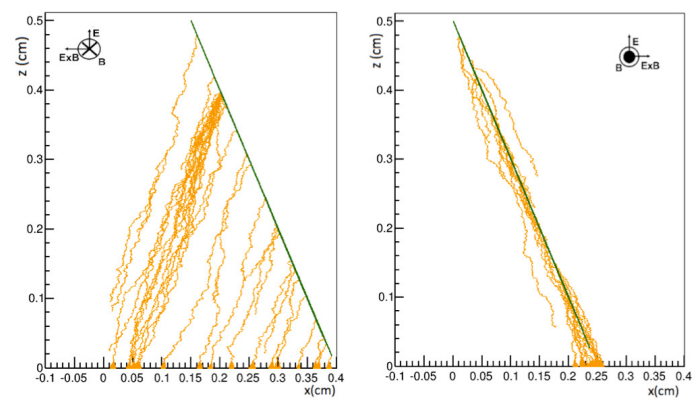

Figure 6. In the magnetic field the drift direction of the ionization electrons is tilted with respect to the electric field direction by the Lorentz angle $\theta_{L}$. Two cases with the magnetic field in opposite directions from Garfield simulation [8].

\section{Test Beam}

Several test-beam campaigns have been performed to test the behaviour of small size resistive micromegas prototypes $\left(10 \times 10 \mathrm{~cm}^{2}\right)$ as well as large prototypes in magnetic fields up to $1 \mathrm{~T}$ using high momentum muon and hadron beams at CERN. Figure 7 shows the MM prototypes inside the "Goliath" dipole magnet at $\mathrm{H} 2$ area at CERN during the December 2014 test beam run. The magnet was able to provide a magnetic field up to $1 \mathrm{~T}$ in two polarities. These studies were performed in order to validate the capability of micromegas chambers to operate and get unbiased tracks in the NSW conditions.
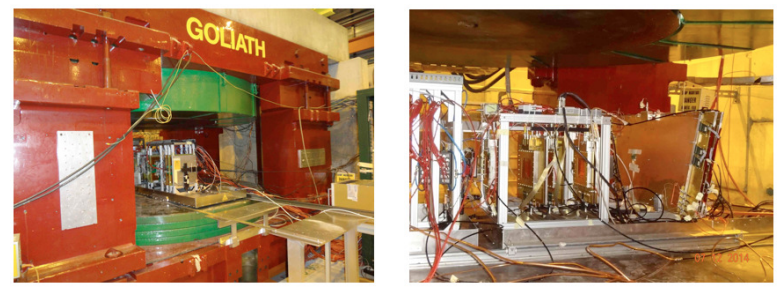

Figure 7. The micromegas chambers installed in the experimental area $\mathrm{H} 2$ at CERN in the Goliath magnet during December 2014 test beam run.

The systematic shifts of the reconstructed position due to the magnetic field can be corrected in two ways: i) by measuring accurately the magnetic field on the NSW with an adequate number of magnetic field sensors, ii) by using the average point measured in a doublet in backto-back configuration, which is free of systematics, as 
demonstrated with simulations.

Figure 8 shows the reconstructed incident angle, the so call effective angle $\lambda$, which does not represent the real track direction because of the Lorentz angle. If the Lorentz angle is known the true incident $\phi$ can be computed. The back-to-back configuration of the doublets is exploited by defining "SuperPoints". Since the shifts of the reconstructed hits in a doublet are equal and in opposite directions, a SuperPoint is defined as the "average" of the two hits. The SuperPoint coordinate $x_{S P}$ is defined by:

$$
x_{S P}=\frac{x_{1}+x_{2}}{2}
$$

where $\mathrm{x} 1$ and $\mathrm{x} 2$ are either centroids or $\mu$ TPC precision coordinates. $x_{S P}$ is provided if the magnetic field (B), the electric field (E) and the drift gap are the same for both chambers in the back-to-back configuration

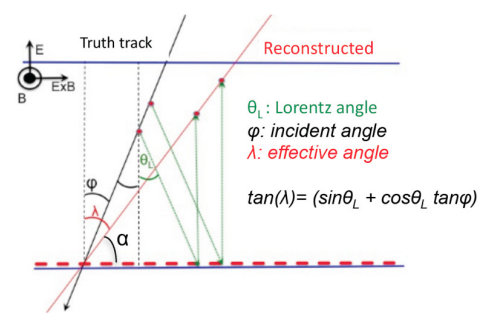

Figure 8. The reconstructed angle of incident $\lambda$, is biased due to the Lorentz angle $\theta_{L}$. Application of the correction formula can give the true incident angle $\phi$.

The values of the Lorentz angle $\theta_{L}$ can be extracted from the data by measuring the shift of the cluster position distributions with respect to no magnetic field case, at the different magnetic field intensities. In Fig. 9 the measured values of $\theta_{L}$ are compared to Garfield values [9].

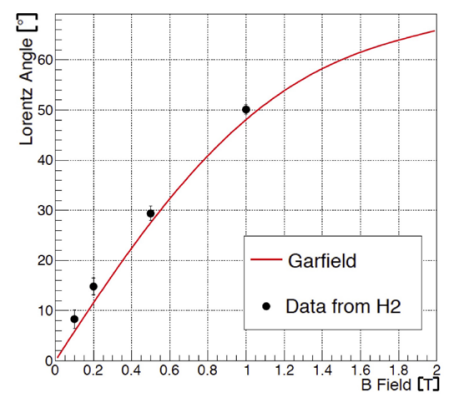

Figure 9. The Lorentz angle as function of the magnetic field (B) calculated with Garfield simulation and experimental points from test beam results.

The component of the drift velocity along E field can be measured, from the distribution of the arrival times of the single clusters knowing the gap size and the applied B field. Knowing the Lorentz angle the Drift Velocity can also be calculated [7].

Figure 10 shows the comparison of the drift velocity with data from the test beam (left) and the compo- nent of drift velocity along the electric field as a function of the magnetic field compared to expectations based on Garfield-Magboltz simulations. Results are consistent with Garfield prediction.
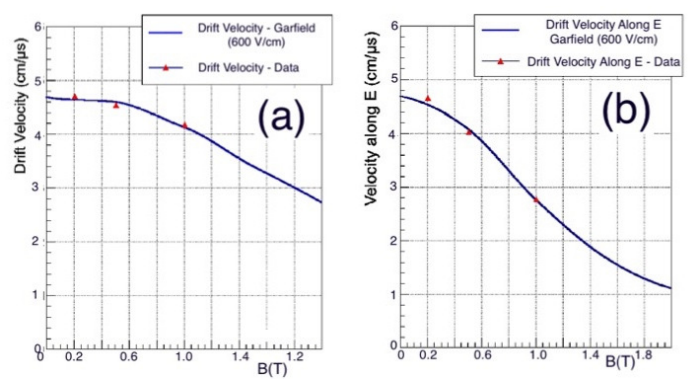

Figure 10. Comparison of the data from test beam with Garfield simulation predictions for the drift velocity (a) and drift velocity along the electric field (b).

\section{Conclusions}

The Micromegas technology chosen for the ATLAS NSW upgrade will enable the Muon Spectrometer to retain its excellent performance. These will be the largest Micromegas detectors ever built for large area chambers in a particle physics experiment and in a high rate environment. Optimised reconstruction techniques have been developed in the context of the test beam studies in order to improve the performance of the Micromegas chambers in magnetic field. Studies inside magnetic field do not show any sign of degraded performance.

\section{References}

[1] LHC Collaboration, The CERN Large Hadron Collider : Accelerator and Experiments, JINST 3 S08001 (2008).

[2] ATLAS Collaboration, The ATLAS Experiment at the CERN Large Hadron Collider, JINST 3 S08003 (2008).

[3] ATLAS Collaboration, ATLAS New Small Wheel TDR, CERN-LHCC-2013-006 ATLAS-TDR-0202013.

[4] Y. Giomataris, P. Rebourgeard, J.P. Robert and G.Charpak, Micro-Pattern Gaseaus Detectors, Nucl. Instrum. and Meth. A376 29-35 (1996) .

[5] M. Bianco, Development of large size Micromegas detectors for the upgrade of the ATLAS experiments, PoS (EPS-HEP 2013) 488 (2013).

[6] T. Alexopoulos, et al., A spark-resistant bulkmicromegas chamber for high-rate applications, Nucl.Instrum.Meth. A640 110-118 (2011).

[7] G. A. Iakovidis, Research and Development in Micromegas Detector for the ATLAS Upgrade, CERNTHESIS-2014-148, (2014).

[8] R. Veenhof, Garfield simulation of the gaseous detectors, urlhttp://garfield.web.cern.ch/garfield/.

[9] K. Ntekas, Design and performance studies of micromegas chambers for the ATLAS muon spectrometer upgrade, ATL-MUON-PROC-2014-011. 